\title{
An Exploration of COVID-19 Impact and Vaccine Hesitancy in Parents of Pediatric Hematopoietic Stem Cell Transplant (HCT) Recipients
}

\author{
Micah Skeens (D) ${ }^{1}$, Parishma Guttoo ${ }^{1}$, Joseph R. Stanek ${ }^{1,2}$, Kimberly Taylor ${ }^{1}$, Erica Stratz ${ }^{1}$, Monica I. Ardura ${ }^{3,4}$ and \\ Hemalatha G. Rangarajan $\mathbb{D}^{1,4 \bowtie}$
}

(c) The Author(s), under exclusive licence to Springer Nature Limited 2022

\begin{abstract}
There is paucity of data on COVID-19 vaccine hesitancy amongst parents of pediatric (age $\leq 17$ years) hematopoietic cell transplant (HCT) recipients. We conducted a cross-sectional study to determined COVID-19 vaccine hesitancy, and COVID-19 impact on family and related distress in this population. A national group $(n=80)$ was recruited via social media (Facebook) from February-May 2021. With vaccine approval for $\geq 12$ years in July 2021, a second group ( $n=37$ ) was recruited locally. Parents completed surveys including the Vaccine Hesitancy Questionnaire and COVID-19 Exposure and Family Impact Scale (CEFIS). Nonparametric statistics were used to analyze results and factors impacting Vaccine Hesitancy Scores (VHS). The majority of parents were non-Hispanic White $(\geq 90 \%)$ and children $\geq 3$ months post-HCT (85\%). Mean CEFIS score (scale 0-60) was 41.11 (SD=8.24), with higher scores indicating negative impact of the pandemic. Mean ( \pm standard deviation) VHS was $2.87( \pm 0.79)$ on a scale of 1-4, with 1 indicating higher and 4 lower hesitancy. Concerns about vaccine related side effects, lower parental age, child age, household income, and education were associated with lower VHS. Receiving reliable information and recommendations by providers was associated with higher VHS. Improving vaccine acceptance in this population is critical in protecting pediatric HCT recipients.
\end{abstract}

Bone Marrow Transplantation (2022) 57:547-553; https://doi.org/10.1038/s41409-022-01587-9

\section{BACKGROUND}

The novel Coronavirus Disease 2019 (COVID-19), caused by severe acute respiratory syndrome coronavirus 2 (SARS-CoV-2), was declared a pandemic by the World Health Organization (WHO) on March 11th, 2020 [1]. The COVID-19 pandemic has had an immense impact on the world population, with almost 285 million cases and 5.4 million deaths to date [2]. Global preventative efforts are necessary to lessen the health and socioeconomic effects of the pandemic [3]. Thus, substantial efforts have focused on developing safe and effective vaccines for COVID-19 [4]. Currently, a total of 60 COVID-19 vaccines are in clinical trial and 170 are in the pre-clinical phase [5]. The US Food and Drug Administration (FDA) and European Medicines Agency approved the use of a COVID-19 vaccine in adolescents (12 and older) in May 2021 [6, 7]. At the time of this report on October 26th, 2021, the FDA advisory panel, after reviewing the data on Pfizer's COVID-19 vaccine in children aged 5-11 years, have recommended emergency use in this age group. Therefore, it is anticipated that most children will be eligible to receive the vaccine in 2022. The American Society of Hematology (ASH) and the American Society of Transplantation and Cellular Therapy (ASTCT) have recommended all hematologic cancer patients, including recipients of hematopoietic stem cell transplant $(\mathrm{HCT})$ be given the COVID-19 vaccine $\geq 3$ months following their stem cell transplant [8]. It is known that HCT recipients may not mount an adequate response to vaccines [9]. The CDC also recommends booster in individuals who have received a HCT within the previous two years or those currently taking immunosuppressive medications.

Despite vast efforts to achieve COVID-19 vaccination for the majority of the population, including children and vulnerable populations, a key component to the success of approved and prospective COVID-19 vaccines is vaccine hesitancy [5]. Vaccine hesitancy has been defined as delay in acceptance, reluctance, or refusal of vaccination [10]. Historically, vaccine hesitancy has been designated by the World Health Organization as 1 of the 10 leading threats to global health, and is not uncommon. One in 15 parents in the United States are hesitant about routine childhood vaccinations and 1 in 4 are hesitant about influenza vaccines [11]. Specific to the COVID-19 vaccine, large variability in COVID-19 vaccine acceptance rates have been found worldwide [5]. A recent survey reported $30 \%$ of adults with cancer were unsure or had no intent to receive the COVID-19 vaccine [12]. In another report, approximately one third of childhood cancer survivor parents expressed hesitancy in vaccinating their survivors against COVID-19 [13]. Similarly, in a survey of 342 adolescents and young adult cancer survivors (15-39 years), 37\% were vaccine hesitant [14]. Determining the degree of vaccine hesitancy and distinguishing other reasons for under-vaccination is essential

\footnotetext{
'Division of Pediatric Hematology, Oncology, Blood and Marrow Transplant, Nationwide Children's Hospital, Columbus, OH, USA. ${ }^{2}$ Biostatistics Resource at Nationwide Children's Hospital, Columbus, OH, USA. ${ }^{3}$ Divisions of Infectious Diseases and Host Defense, Nationwide Children's Hospital, Columbus, OH, USA. ${ }^{4}$ Department of Pediatrics, The Ohio State University, Columbus, OH, USA. ${ }^{凶}$ email: hemalatha.rangarajan@nationwidechildrens.org
}

Received: 26 November 2021 Revised: 13 January 2022 Accepted: 14 January 2022

Published online: 24 January 2022 
to develop interventions that address substandard vaccine uptake [15].

To our knowledge, there is currently little information on vaccine hesitancy amongst parents of children after HCT. Therefore, we conducted a prospective descriptive study to explore baseline vaccine hesitancy and investigate potential factors associated with vaccine hesitancy in this high-risk population. Additionally, we assessed the effects (impact, distress) of the COVID-19 pandemic on parents of HCT recipients and the correlation to vaccine hesitancy.

\section{METHODS}

This paper presents a cross-sectional analysis of COVID-19 vaccine hesitancy among parents of children who have undergone a hematopoietic stem cell transplant. Approval was obtained from our local institutional review board to conduct this prospective study. Participant eligibility included English speaking parents of children, aged 0 to $\leq 17$ years of age, with a history of HCT. Consent and assent were implied by completion of the anonymous survey.

\section{Recruitment}

Parents were recruited in two independent groups. Initially, in a larger study examining COVID-19 impact and vaccine hesitancy in cancer families, recruitment was conducted in partnership with Momcology ${ }^{\circledast}$, a nonprofit pediatric cancer community-based organization using a pay-perclick Facebook ad campaign. Momcology ${ }^{\circledR}$ participates in communityengagement efforts to connect parents to IRB-approved research studies that will benefit the needs of its community [16]. Recruitment occurred from February 2021 until May 2021 and ended prior to approval of the vaccine in adolescents greater than 16 years of age. The Facebook advertisement included a black and white picture of a child with cancer (Supplementary Fig. 1). Parents utilized a hyperlink to complete a four-item eligibility screening questionnaire; eligible parents were then directed to complete electronic measures via REDCap. This approach was chosen due to social and physical distancing orders and previous research has shown online and mail surveys produce equivalent results $[17,18]$. Following vaccine approval in children over 12 years of age, a second group of parents was recruited locally, at our center, to further evaluate vaccine hesitancy following approval in older children and adults. These families were recruited over an 8-week span in June and July 2021. The parents were approached for participation in the following ways: (1) in person during a clinic visit, (2) via email (if available), or (3) using the messaging function within the electronic medical record (if enrolled). The study information sheet included the purpose, benefits, risks, and anticipated time to complete the survey.

\section{Measures}

Demographic characteristics. Data were collected from the parent about themselves and partner (if applicable), including number of children, sex, race, ethnicity, marital status, geographic location, income, employment status, occupation, and COVID-19 exposure. Parents were also asked about the participating child's age, grade, sex, race, ethnicity, underlying diagnosis, and age at transplant.

COVID-19 exposure and family impact scale (CEFIS). This standardized measure assesses the exposure to events and impact of the COVID-19 pandemic on families. Part 1, measuring exposure is described elsewhere and was not used in this study [19]. Part 2 is comprised of 12 items

Table 1. Parent demographic characteristics $(N=113)$.

\begin{tabular}{|c|c|c|c|c|}
\hline & All participants $N=113$ & National cohort $N=76$ & Local cohort $N=37$ & $p$-value ${ }^{1}$ \\
\hline Parent Age in years & $40.7(7.0)$ & $42.1(6.5)$ & $37.9(7.4)$ & 0.0014 \\
\hline \multicolumn{5}{|l|}{ Sex } \\
\hline Female & 104 (93\%) & 71 (95\%) & $33(89 \%)$ & 0.44 \\
\hline \multicolumn{5}{|l|}{ Race } \\
\hline White & $101(90 \%)$ & $70(92 \%)$ & 31 (86\%) & 0.33 \\
\hline \multicolumn{5}{|l|}{ Ethnicity } \\
\hline Hispanic & $4(3 \%)$ & $4(5 \%)$ & $0(-)$ & \multirow[t]{2}{*}{0.30} \\
\hline Non-Hispanic & $108(96 \%)$ & 72 (95\%) & $36(100 \%)$ & \\
\hline \multicolumn{5}{|l|}{ Education } \\
\hline High school or less & $18(16 \%)$ & $14(18 \%)$ & $4(11 \%)$ & 0.0309 \\
\hline$\$ 25,001-\$ 50,000$ & $19(17 \%)$ & $12(16 \%)$ & $7(19 \%)$ & \multirow{5}{*}{0.83} \\
\hline$\$ 50,001-\$ 75,000$ & $19(17 \%)$ & $13(17 \%)$ & $6(17 \%)$ & \\
\hline$\$ 75,001-100,000$ & $22(20 \%)$ & $15(20 \%)$ & $7(19 \%)$ & \\
\hline$\$ 100,001-\$ 150,000$ & $26(23 \%)$ & $20(27 \%)$ & $6(17 \%)$ & \\
\hline More than $\$ 150,000$ & $16(14 \%)$ & $9(12 \%)$ & $7(19 \%)$ & \\
\hline \multicolumn{5}{|l|}{ Current Employment Status } \\
\hline Full-time (>30 h/week) & 49 (43\%) & $29(38 \%)$ & $20(54 \%)$ & \multirow[t]{3}{*}{0.21} \\
\hline Part-time (<30 h/week) & $24(21 \%)$ & $19(25 \%)$ & $5(14 \%)$ & \\
\hline Unemployed & 40 (35\%) & 28 (37\%) & 12 (32\%) & \\
\hline
\end{tabular}

All data are presented as $N(\%)$ or median (range).

${ }^{1} P$-values result from Chi-square or Fisher's exact tests or Wilcoxon rank sum tests.

Note: Some items may not sum to the total $N$ due to some missing values. 
Table 2. Child demographic characteristics $(N=113)$.

\begin{tabular}{|c|c|c|c|c|}
\hline & All participants $N=113$ & National cohort $N=76$ & Local cohort $N=37$ & $p$-value ${ }^{2}$ \\
\hline Child Age in years & $9.2(4.6)$ & $9.8(4.8)$ & $8.0(3.9)$ & 0.07 \\
\hline \multicolumn{5}{|l|}{ Sex } \\
\hline Male & $60(53 \%)$ & $40(53 \%)$ & $20(54 \%)$ & \multirow[t]{2}{*}{0.89} \\
\hline Female & $53(47 \%)$ & $36(47 \%)$ & $17(46 \%)$ & \\
\hline \multicolumn{5}{|l|}{ Race } \\
\hline White & $100(89 \%)$ & $68(89 \%)$ & 32 (89\%) & 0.99 \\
\hline \multicolumn{5}{|l|}{ Ethnicity } \\
\hline Hispanic & $5(4 \%)$ & $4(5 \%)$ & $1(3 \%)$ & \multirow[t]{2}{*}{0.99} \\
\hline Non-hispanic & $108(96 \%)$ & 72 (95\%) & $36(97 \%)$ & \\
\hline \multicolumn{5}{|l|}{ Primary diagnosis } \\
\hline Leukemia & 35 (31\%) & $29(38 \%)$ & $6(16 \%)$ & 0.0010 \\
\hline 3 months- 1 year & $17(15 \%)$ & $14(18 \%)$ & $3(8 \%)$ & \multirow{2}{*}{0.30} \\
\hline$>1$ year & $79(70 \%)$ & $50(66 \%)$ & $29(78 \%)$ & \\
\hline \multicolumn{5}{|l|}{ BMT characteristics ${ }^{1}$} \\
\hline Age at BMT & $4(<1-15)$ & NA & $4(<1-15)$ & - \\
\hline Autologous & $17(46 \%)$ & NA & $17(46 \%)$ & - \\
\hline Allogenic & $17(46 \%)$ & NA & $17(46 \%)$ & - \\
\hline Unknown BMT type & $3(8 \%)$ & NA & $3(8 \%)$ & - \\
\hline CAR-T Therapy & 4/31 (13\%) & NA & 4/31 (13\%) & - \\
\hline
\end{tabular}

measuring the impact of COVID-19 (scores range from 0 to 60 ), such as effects on parenting, ability to care for children, and physical well-being. Ten items were rated on a five-point Likert scale (modified from the original four points to include no change) indicating the degree to which COVID-19 affected each area. Two additional items measured parental and child distress on a 10-point scale, with higher scores indicating a more negative impact. Higher scores were indicative a more negative impact of COVID-19 pandemic on surveyed individuals.

Vaccine hesitancy questionnaire (VHS). This questionnaire was a modified version of Kempe's vaccine hesitancy questionnaire originally adapted from the WHO Vaccine Hesitancy Survey. The VHS survey is psychometrically validated and encompasses ten items with Likert responses, including dimensions of vaccine confidence and vaccine risks [20]. The modified version includes eight items and is on a four-point Likert scale rather than a five-point response scale due to removal of the "neutral" option given evidence that omitting the "neutral" option decreases the potential for socially desirable responding [21]. The wording was further modified to address COVID-19 vaccination rather than general childhood or influenza vaccines. Individual VHS items were given a point value of 1 to 4 with a score of 1 given to responses indicating vaccine hesitancy (e.g., choosing "Strongly disagree" that the vaccine is effective, beneficial to their child, able to protect their child from illness, and that the information about the vaccine is reliable and trustworthy) and a score of 4 indicating vaccine acceptance (e.g., choosing "Strongly agree" for the vaccine is effective, beneficial, protective, etc.) Using a subset of six survey items, a composite VHS score was calculated by averaging each parents' responses to have an overall measurement of their vaccine hesitancy. These six survey items included questions regarding COVID-19 vaccine efficacy, benefit, and protection; the reliability and trustworthiness of information regarding COVID-19 vaccines; and degree of concern for vaccine side effects for themselves and their children. The local group survey contained additional questions on type of transplant (autologous vs allogeneic), and four additional questions to further explore COVID-19 specific vaccine hesitancy.

\section{Statistical analysis}

Survey responses were summarized descriptively: frequency and percentage for qualitative variables and median and range or mean and standard deviation (SD) for quantitative variables. Comparisons of parent and child demographic information were compared between the national and local groups using nonparametric methods: chi-square or Fisher's exact tests and Wilcoxon rank-sum tests. Kruskal-Wallis and Wilcoxon rank-sum tests were used to compare vaccine hesitancy scores between parent groups, such as income, education, and other characteristics. Spearman's correlation coefficients were computed to assess the correlation between vaccine hesitancy and age and CEFIS scores. $P$-values were two-sided and those $<0.05$ were considered statistically significant. These comparisons were exploratory in nature but may inform future research about what characteristics are associated with vaccine hesitancy. Statistical analysis was completed using the base $\mathrm{R}$ statistical package ( $\mathrm{R}$ Foundation for Statistical Computing, Vienna, Austria).

\section{RESULTS}

\section{Participant characteristics}

A total of 113 parents completed the survey including 76 from the national social media campaign and 37 locally. Parents were mostly White $(n=101,90 \%)$, Non-Hispanic $(n=108,96 \%)$, with at least some college level of education $(n=94,83 \%)$ and a median age of 40.7 years. (Table 1 ). Household income was equally distributed in both national and local groups. The characteristics of HCT recipients are shown in Table 2 . Children were also predominantly White $(89 \%, n=100)$ and Non-Hispanic $(96 \%, n=108)$, male $(53 \%$, 


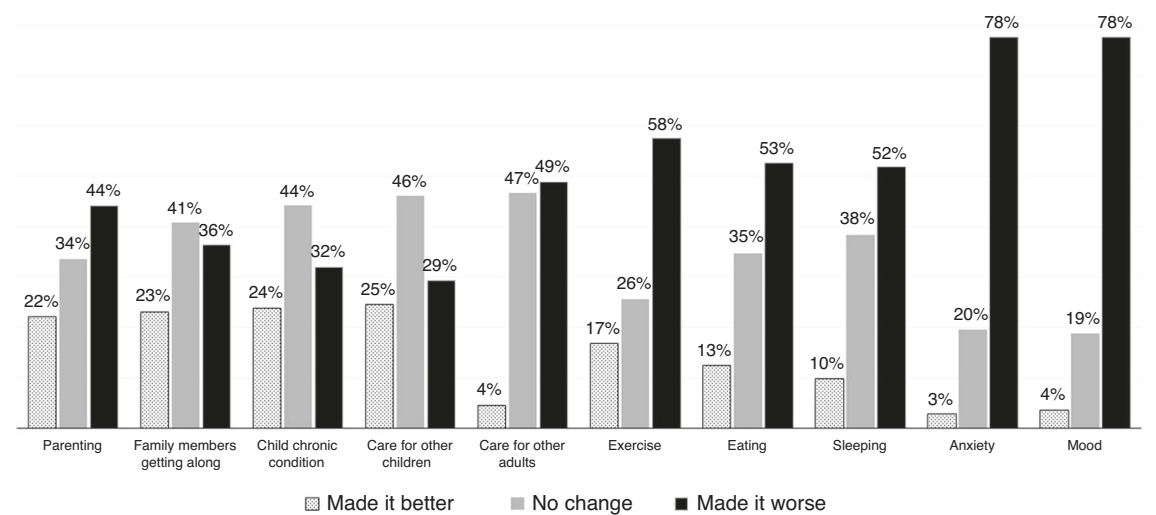

Fig. 1 Individual CEFIS item responses. The summarized CEFIS Impact scale responses from the total study group ( $n=113$ ). Responses were categorized as made it better, no change and made it worse.

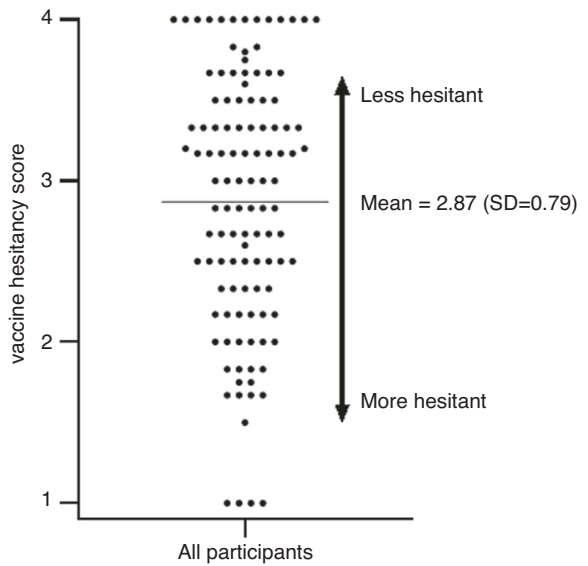

Fig. 2 Vaccine Hesitancy Score (VHS) of the Whole Study Group $(n=113)$. The Mean ( \pm Standard Deviation) Vaccine Hesitancy Score (VHS) of the whole study group was $2.87( \pm 0.79)$ with 1 indicating higher vaccine hesitancy and 4 lower vaccine hesitancy.

$n=60)$ with a median age of 9.33 years $(S D=4.61)$. Most children were $\geq 3$ months from transplant $(n=96,85 \%)$. The most common primary diagnosis was solid tumors $(n=54,48 \%)$ followed by leukemia $(n=35,31 \%)$ and non-malignant disorders $(n=24,21 \%)$. In the local group, there were an equal number of autologous $(46 \%, n=17)$ and allogenic participants $(46 \%, n=17)$, including four Chimeric antigen Receptor T cell (CAR-T) therapy recipients. Compared to the national group, the parents in the local group were younger on average (37.9 years vs 42.1 years; $p=0.0014$ ) and had a greater proportion of the children with a non-malignant disorder as the primary diagnosis ( $11 \%$ vs $41 \% ; p=0.001)$.

\section{COVID-19 family impact (CEFIS) and COVID distress}

The average COVID impact and distress score was $41.11(S D=8.24)$ on a scale of 0 to 60 . The CEFIS Impact scale responses from the total sample are summarized in Fig. 1. Most parents reported that COVID-19 worsened anxiety (77.7\%), mood (77.7\%), parenting (44.2\%), and their ability to care for older adults (48.9\%). Parental physical wellbeing (exercise (57.5\%), eating (52.7\%), and sleep $(51.8 \%)$ also deteriorated due to the COVID-19 pandemic. However, the ability to care for one's sick child (44.2\%) and other children $(46.1 \%)$ was not as affected. Parents reported a significantly higher mean distress score for themselves vs their children: 6.32 (SD= $2.53)$ vs $5.55(\mathrm{SD}=2.81)$ (t $(224)=2.146, p=0.033)$. The mean distress scores were also higher in the national group for both parent (6.82 vs 5.29) (t $(111)=3.12, p=0.0023)$ and children (6.22 vs 4.18$)$ ( $\mathrm{t}(111)=3.85, p=0.0002)$ than in the local group.

\section{Vaccine hesitancy}

The mean vaccine hesitancy score (VHS) of the entire study group was $2.87(S D=0.79)$ on a scale of 1 to 4 (Fig. 2), with 1 indicating higher vaccine hesitancy and 4 lower vaccine hesitancy. Questions that were associated with lower scores (higher vaccine hesitancy) included concerns for vaccine side effects both in the child $(M=$ $2.08, S D=1.05)$ and parent $(M=2.51, S D=1.10)$ (Fig. 3). On the other hand, higher scores (less vaccine hesitancy) were associated with questions regarding access to reliable and trustworthy vaccine related information $(M=3.26, S D=0.89)$. Results for the additional vaccine questions administered to the local group are shown in Fig. 3. This group was less hesitant to receiving the vaccine and a booster dose if recommended by the child's health care provider.

\section{Patient and parent characteristics associated with vaccine hesitancy}

(Table 3) We observed a weak positive correlation between parent age and vaccine hesitancy $\left(r_{\mathrm{s}}=0.34 ; p=0.0003\right)$, inferring younger parents were more likely to be vaccine hesitant. Similarly, increased vaccine hesitancy was also noted with parents of younger children $\left(r_{\mathrm{s}}=0.25 ; p=0.0086\right)$. Vaccine hesitancy was greater among the lower income groups (Table 3). Parents with at least a bachelor's degree were less vaccine hesitant $(M=3.02, S D=0.73)$ compared to those with some college $(M=2.49, S D$ 0.76) or high school education only $(M=2.73, S D=0.90)$ (Kruskal-Wallis, $p=0.0119$ ). Among parents who were not likely to be vaccinated, lower VHS (more vaccine hesitancy) was observed (Table 3, Supplementary Fig. 2). When exploring associations between other factors and vaccine hesitancy, there was no statistically significant association between vaccine hesitancy and employment status $(p=0.13)$, posttransplant duration $(p=0.93)$, or primary diagnosis $(p=0.63)$. Parent-reported CEFIS impact score was not correlated with vaccine hesitancy $\left(r_{s}=0.05, p=0.60\right)$. Additionally, neither parent's $(r=0.05$, $p=0.63)$ nor child's $\left(r_{s}=0.09, p=0.35\right)$ distress scores were correlated with vaccine hesitancy.

\section{DISCUSSION}

COVID-19 vaccine is of critical importance in HCT recipients as they are at increased risk of mortality and morbidity following a SARSCoV-2 infection secondary to delayed immune reconstitution, need for ongoing immunosuppression and possible suboptimal responses to vaccinations [22]. There is limited knowledge on the effects of the COVID-19 pandemic on families of children who have undergone 
Survey questions asked to both groups (facebook and local)

\begin{tabular}{|l|l|}
\hline Receiving reliable and trustworthy information & $3.26( \pm 0.9)$ \\
\hline \hline COVID-19 vaccine is effective & $3.19( \pm 0.9)$ \\
\hline \hline COVID-19 vaccine is a good way to protect my child from illness & $3.16( \pm 1.0)$ \\
\hline \hline I believe the COVID-19 vaccine will be beneficial to my child & $2.51( \pm 1.1)$ \\
\hline \hline I am concerned about COVID-19 vaccine side effects/safety for myself \\
\hline \hline I am concerned about COVID-19 vaccine side effects/safety for my child $2.08( \pm 1.0)$ \\
\hline
\end{tabular}

Survey questions asked to only the local group

\begin{tabular}{|l|l|}
\hline I will follow my child's health care providers recommendation about COVID-19 vaccine & $3.22( \pm 0.9)$ \\
\hline \hline If recommended by my provider, I will accept the booster vaccine for my child & $3.06( \pm 1.0)$ \\
\hline \hline The COVID-19 vaccine contributes to herd immunity & $2.97( \pm 1.1)$ \\
\hline \hline The COVID-19 vaccine is important to my health & $2.76( \pm 1.1)$
\end{tabular}

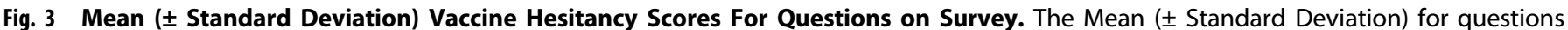
associated with lower and higher Vaccine Hesitancy Scores (VHS) on the survey.

Table 3. Impact of demographic variables with vaccine hesitancy scores (VHS).

\begin{tabular}{|c|c|c|c|c|}
\hline & $\begin{array}{l}\text { Study } \\
\text { Group (N) }\end{array}$ & Mean VHS & SD & $p$-value \\
\hline Overall & 113 & 2.87 & 0.79 & \\
\hline \multicolumn{5}{|l|}{ Diagnosis } \\
\hline Leukemia & 35 & 2.78 & 0.79 & \multirow[t]{3}{*}{0.63} \\
\hline Solid tumors & 54 & 2.87 & 0.80 & \\
\hline Non-malignant & 24 & 2.98 & 0.77 & \\
\hline \multicolumn{5}{|l|}{ Education level } \\
\hline High school or less & 18 & 2.73 & 0.90 & \multirow[t]{3}{*}{0.0119} \\
\hline Some college & 24 & 2.49 & 0.76 & \\
\hline Bachelor's or greater & 70 & 3.02 & 0.73 & \\
\hline \multicolumn{5}{|l|}{ Income } \\
\hline$\leq \$ 25,000$ & 9 & 2.72 & 0.62 & \multirow[t]{6}{*}{0.0016} \\
\hline$\$ 25,000-50,000$ & 19 & 2.39 & 0.65 & \\
\hline$\$ 50,000-75,000$ & 19 & 2.66 & 0.57 & \\
\hline$\$ 75,000-100,000$ & 22 & 2.87 & 1.00 & \\
\hline$\$ 100,000-150,000$ & 26 & 3.11 & 0.69 & \\
\hline$>\$ 150,000$ & 16 & 3.34 & 0.80 & \\
\hline \multicolumn{5}{|l|}{ Parent self-vaccine status } \\
\hline Have/will be vaccinated & 64 & 3.32 & 0.54 & \multirow[t]{4}{*}{$<0.0001$} \\
\hline Very likely & 9 & 2.93 & 0.68 & \\
\hline Will consider it & 20 & 2.35 & 0.52 & \\
\hline Not likely & 18 & 1.82 & 0.47 & \\
\hline \multicolumn{5}{|l|}{ Employment status } \\
\hline Full-time & 49 & 2.98 & 0.76 & \multirow[t]{3}{*}{0.13} \\
\hline Part-time & 24 & 2.98 & 0.70 & \\
\hline Unemployed & 40 & 2.67 & 0.85 & \\
\hline \multicolumn{5}{|l|}{ Post-transplant time } \\
\hline$<3$ months & 17 & 2.95 & 0.76 & \multirow[t]{3}{*}{0.93} \\
\hline 3 months-1 year & 17 & 2.87 & 0.82 & \\
\hline$>1$ year & 79 & 2.85 & 0.80 & \\
\hline
\end{tabular}

$P$-values result from Kruskal-Wallis tests.

HCT. With the approval of COVID-19 vaccines for children $\geq 5$ years, it is important to ascertain the baseline vaccine hesitancy in caregivers of pediatric HCT recipients, which is currently unknown. Therefore, we studied both the impact of the COVID-19 pandemic, and vaccine hesitancy in a large sample of parents of pediatric HCT recipients. We observed higher COVID-related impact and distress scores, indicative of the detrimental impact the COVID-19 pandemic in our study population. Although the mean COVID VHS was 2.87 , concern about vaccine related side effects was a major contributor to hesitancy. Alternatively, receiving reliable, trustworthy information and recommendation by providers contributed to less vaccine hesitancy.

The mean CEFIS score was 41.1 and we observed a higher COVID-related distress in parents than reported in children. Consistent with other studies, we observed negative effects of the pandemic on mood, anxiety, ability to care for others, and physical well-being $[19,23,24]$. Contrarily, a recent study in adult HCT recipients found no difference in distress, fatigue, or quality of life before or during the pandemic [25]. However, a large body of literature suggests the mental health effects of the pandemic are significant among parents and children [23, 26-29].

In this US group of parents of children post-HCT, overall COVID19 vaccine hesitancy was low. One might expect less vaccine hesitancy if families are provided education about the increased risk of COVID-19 in their vulnerable children. A recent study utilizing a provider-delivered webinar demonstrated increased COVID-19 vaccine enthusiasm in adults with cancer [16]. Additionally, in our study sample parents expressed receiving timely and reliable information as well as provider recommendations decreased vaccine hesitancy. This strongly supports the need for timely, accurate information regarding the short-term risks and the long-term benefits of the COVID-19 vaccination from health care providers.

Parents in our study with lower income, less education, younger age, and younger children were more vaccine hesitant. These associations are similar to previous studies in which lower income and education level predicted hesitancy about routine childhood and influenza vaccines [11]. Age of the child was an important contributor to hesitancy within this cohort, similar to a recent study of childhood cancer survivors in which parents were more willing to vaccinate their survivor if they were of older age [13]. Many of our observations also mirror the results of the COVID States Project Report\#45 on COVID vaccine hesitancy and resistance [30]. In this large 50 state online survey of 19,789 individuals' ( $\geq 18$ years) with or without children under 18 years, parents were found to be more vaccine hesitant and resistant than non-parents across all socioeconomic and demographic groups, largely driven by younger mothers who were more vaccine resistant than younger women who were not mothers. Parents' willingness to vaccinate their children closely matched their willingness to vaccinate themselves. Parents, without a 4-year degree and income of $<\$ 75,000$ per year were more vaccine 
hesitant than their counterparts with a 4-year college degree or income $>\$ 75,000$. In our study, we observed concern for side effects and the safety of the vaccine also contributed to increased vaccine hesitancy. This is not surprising, since most literature suggests adverse effects and safety concerns remain the largest determinants of whether parents vaccinate their children [11,31].

To our knowledge, this is one of the first studies to examine vaccine hesitancy in parents of children post-HCT; however, this study should be considered in the context of several limitations. First, multiple methods of data collection including the use of social media for recruitment could introduce ascertainment bias and restricts participation of parents without social media access. Due to our study design, we are unable to determine the characteristics of non-respondents. Parents were primarily White, non-Hispanic mothers. The lack of diversity within our sample could bias the results and decrease generalizability. It also draws attention to potential barriers diverse populations face in access to information and research participation. Continued research is needed to understand the development and resolution of vaccine hesitancy in unique vulnerable populations. Inclusion of diverse families who may have additional challenges due to health disparities, such as higher rates of COVID infection and mortality, greater COVID-related impact, and less access to the vaccine is important. Notably in the COVID-19 States Project survey, 75\% of African-American parents are vaccine hesitant or resistant [30]. Recruitment of a large majority of participants in this study, occurred in the early days of vaccination when the vaccine was not approved for children or adolescents. Vaccine hesitancy may decrease as approval for younger ages is achieved and as time from vaccination increases with few adverse reactions.

\section{CONCLUSION}

Results from this study may inform targeted interventions to increase COVID-19 vaccination rates in children post-HCT. Potential interventions could include use of social media to provide accurate timely information while dispelling myths, as well improved access to FDA/CDC data regarding safety and efficacy of vaccines. Although COVID-19 vaccines development has progressed at rapid speed, a vaccine not given does not provide benefit. Vaccine confidence and hesitancy influences vaccine acceptance. Decreasing hesitancy rates in parents of children post-HCT with special attention to those of lower income, less education, younger age and with children of younger age, is needed to ensure all children post-HCT are protected. Transplant teams are urged to offer COVID-specific education and information about the vaccine, which has been shown to be effective in other vaccine hesitancy research.

\section{REFERENCES}

1. World Health Organization. WHO Director-General's opening remarks at the media briefing on COVID-19-11 March 2020. https://www.who.int/directorgeneral/speeches/detail/who-director-general-s-opening-remarks-at-the-mediabriefing-on-covid-19---11-march-2020.

2. University JH. Center for Systems Science and Engineering. Coronavirus Resource Center. Available at https://coronavirus.jhu.edu/.

3. Calina D, Docea AO, Petrakis D, Egorov AM, Ishmukhametov AA, Gabibov AG, et al. Towards effective COVID-19 vaccines: Updates, perspectives and challenges. Int J Mol Med. 2020;46:3-16.

4. Conte C, Sogni F, Affanni P, Veronesi L, Argentiero A, Esposito S. Vaccines against coronaviruses: the state of the art. Vaccines. 2020;8:309.

5. Sallam M. COVID-19 vaccine hesitancy worldwide: a concise systematic review of vaccine acceptance rates. Vaccines. 2021;9:160.

6. Zou X, Cao B. COVID-19 vaccines for children younger than 12 years: are we ready? Lancet Infect Dis. 2021;21:1614-15.

7. Frenck RW, Klein NP, Kitchin N, Gurtman A, Absalon J, Lockhart S, et al. Safety, immunogenicity, and efficacy of the BNT162b2 Covid-19 vaccine in adolescents. N. Engl J Med. 2021;385:239-50.
8. Ali H, Ngo D, Aribi A, Arslan S, Dadwal S, Marcucci G, et al. Safety and tolerability of SARS-CoV2 emergency-use authorized vaccines for allogeneic hematopoietic stem cell transplant recipients. Transplant Cell Ther. 2021;27:938-e1.

9. Redjoul R, Le Bouter A, Parinet V, Fourati S, Maury S. Antibody response after third BNT162b2 dose in recipients of allogeneic HSCT. Lancet Haematol. 2021;8: e681-3.

10. Soares P, Rocha JV, Moniz M, Gama A, Laires PA, Pedro AR, et al. Factors associated with COVID-19 vaccine hesitancy. Vaccines. 2021;9:300.

11. Kempe A, Saville AW, Albertin C, Zimet G, Breck A, Helmkamp L, et al. Parental hesitancy about routine childhood and influenza vaccinations: a national survey. Pediatrics. 2020;146:e20193852.

12. Kelkar AH, Blake JA, Cherabuddi K, Cornett H, McKee BL, Cogle CR, editors. Vaccine Enthusiasm and Hesitancy in Cancer Patients and the Impact of a Webinar. Healthcare; 2021: Multidisciplinary Digital Publishing Institute.

13. Wimberly CE, Towry L, Davis E, Johnston EE, Walsh KM. SARS-CoV-2 vaccine acceptability among caregivers of childhood cancer survivors. Pediatr Blood Cancer. 2021:e29443.

14. Waters AR, Kepka D, Ramsay JM, Mann K, Vaca Lopez PL, Anderson JS, et al. COVID-19 vaccine hesitancy among adolescent and young adult cancer survivors. JNCl Cancer Spectr. 2021;5:pkab049.

15. MacDonald NE. Vaccine hesitancy: Definition, scope and determinants. Vaccine. 2015;33:4161-4.

16. Santacroce SJ, Kneipp SM. Influence of pediatric cancer-related financial burden on parent distress and other stress-related symptoms. Pediatr Blood Cancer. 2020;67:e28093.

17. Deutskens E, De Ruyter K, Wetzels M. An assessment of equivalence between online and mail surveys in service research. J Serv Res. 2006;8:346-55.

18. Walter SL, Seibert SE, Goering D, O'Boyle EH. A tale of two sample sources: Do results from online panel data and conventional data converge? J Bus Psychol. 2019;34:425-52.

19. Kazak AE, Alderfer M, Enlow PT, Lewis AM, Vega G, Barakat L, et al. COVID-19 exposure and family impact scales: factor structure and initial psychometrics. J Pediatr Psychol. 2021;46:504-13.

20. Luyten J, Bruyneel L, van Hoek AJ. Assessing vaccine hesitancy in the UK population using a generalized vaccine hesitancy survey instrument. Vaccine. 2019;37:2494-501.

21. Chyung SY, Roberts K, Swanson I, Hankinson A. Evidence-based survey design: The use of a midpoint on the Likert scale. Perform Improvement. 2017;56:15-23.

22. Sharma A, Bhatt NS, St Martin A, Abid MB, Bloomquist J, Chemaly RF, et al. Clinical characteristics and outcomes of COVID-19 in haematopoietic stem-cell transplantation recipients: an observational cohort study. Lancet Haematol. 2021;8:e185-e93.

23. Patrick SW, Henkhaus LE, Zickafoose JS, Lovell K, Halvorson A, Loch S, et al. Well-being of parents and children during the COVID-19 pandemic: a national survey. Pediatrics. 2020;146:e2020016824.

24. Gassman-Pines A, Ananat EO, Fitz-Henley J, 2nd. COVID-19 and parent-child psychological well-being. Pediatrics. 2020;146.

25. Amonoo HL, Topping CEW, Clay MA, Reynolds MJ, Rice J, Harnedy LE, et al. Distress in a pandemic: association of the coronavirus disease-2019 pandemic with distress and quality of life in hematopoietic stem cell transplantation. Transplant Cell Ther. 2021;27:1015-e1.

26. Raviv T, Warren CM, Washburn JJ, Kanaley MK, Eihentale L, Goldenthal HJ, et al. Caregiver perceptions of children's psychological well-being during the COVID19 pandemic. JAMA Netw Open. 2021;4:e2111103.

27. Ammar A, Mueller $P$, Trabelsi $K$, Chtourou H, Boukhris $O$, Masmoudi $L$, et al. Psychological consequences of COVID-19 home confinement: The ECLB-COVID19 multicenter study. PLoS One. 2020;15:e0240204.

28. Salari N, Hosseinian-Far A, Jalali R, Vaisi-Raygani A, Rasoulpoor S, Mohammadi M, et al. Prevalence of stress, anxiety, depression among the general population during the COVID-19 pandemic: a systematic review and meta-analysis. Globalization Health. 2020;16:1-11.

29. Lades LK, Laffan K, Daly M, Delaney L. Daily emotional well-being during the COVID-19 pandemic. Br J Health Psychol. 2020;25:902-11.

30. Simonson M, Matthew A, Lazer D, Ognyanova K, Gitomer A, Perlis $\mathrm{RH}$, et al. THE STATE OF THE NATION: A 50-STATE COVID-19 SURVEY. Report \#45: COVID19 Vaccine Hesitancy and Resistance Among Parents www.covidstates.org/reports or at https://osf.io/e95bc/2021.

31. Olusanya OA, Bednarczyk RA, Davis RL, Shaban-Nejad A. Addressing parental vaccine hesitancy and other barriers to childhood/adolescent vaccination uptake during the coronavirus (COVID-19) pandemic. Front Immunol. 2021;12:855.

\section{AUTHOR CONTRIBUTIONS}

MS and HGR were responsible for designing the study, analyzing the data, interpreting results, writing and editing of the manuscript. PG was responsible for 
collection of data, performing preliminary statistical analysis and interpretation of results, writing and editing of the manuscript. JRS was performed statistical analysis. ES and KT contributed to data collection. interpretation of the results and edited the manuscript. MIA assisted with study design and editing of the final manuscript.

\section{FUNDING}

Nationwide Children's Hospital Intramural Funds.

\section{COMPETING INTERESTS}

The authors declare no competing interests.

\section{ADDITIONAL INFORMATION}

Supplementary information The online version contains supplementary material available at https://doi.org/10.1038/s41409-022-01587-9.

Correspondence and requests for materials should be addressed to Hemalatha G. Rangarajan.

Reprints and permission information is available at http://www.nature.com/ reprints

Publisher's note Springer Nature remains neutral with regard to jurisdictional claims in published maps and institutional affiliations. 